\title{
Recepción de la información política televisada en niños regiomontanos durante la campaña presidencial del 2006
}

\author{
ALEJANDRA RODRÍGUEZ ESTRADA ${ }^{1}$ \\ Y CARLOS MUÑIZ MURIEL2
}

Este artículo reporta, a partir de la triangulación de las técnicas cuantitativa y cualitativa, el análisis de 588 encuestas y 30 entrevistas a niños de Monterrey, Nuevo León en 2006, con la finalidad de aproximarse al papel que ocupa la política televisada en las opiniones y percepciones de los niños, así como para comprender hasta qué punto es distinto su acercamiento de acuerdo al tipo de escuela a la que pertenecen y del capital cultural heredado de sus padres. La investigación se enfocó en el fenómeno de recepción desde la brecha educativa en nuestro país, a fin de identificar cómo la estructura educativa está también involucrada en la brecha de capacidades interpretativas, reflexivas y críticas.

PALABRAS CLAVE: socialización política, recepción televisiva, niños, elecciones, cultura política.
The article reports the results of the analysis of 588 surveys and 30 interviews to Monterrey's children in 2006, based on the triangulation of quantitative and qualitative techniques. The study intends to achieve a clear approach to the influence of televised politics among kids, as well as to reveal how different is the kid's opinion depending on their kind of school and his/her parent's opinion or inherited cultural baggage. It focuses on the reception phenomenon due to the educative gap in México, in order to learn how the educative structure is involved in this gap of interpretative, reflective and critic capacities towards the promotion of a better citizenship and the equality of power.

KEY WORDS: politic socialization, televisional reception, political culture, elections.

1 Universidad Anáhuac de Xalapa, México.

Correo electrónico: ale0323@gmail.com

2 Instituto Tecnológico y de Estudios Superiores de Monterrey, México.

Correo electrónico: carmuniz@itesm.mx 


\section{Cultura POLÍTiCA EN MÉXICO³}

México es un país que requiere prestar mayor atención a la formación de la cultura política de sus ciudadanos. La Encuesta Nacional de Cultura Política (SEGOB, 2002) levantada en 2001, reveló que 60\% de los jóvenes ignoraba su papel respecto a los problemas que trata de resolver el gobierno y una gran mayoría de los jóvenes encuestados (82\%) reportaba que nunca había trabajado de manera formal o informal con otras personas para tratar de resolver problemas de su comunidad. En el 2001, los datos de la Encuesta Nacional de Cultura Política (ENCUP) sugirieron que en México existía un escaso interés en la política entre la población mayor de 18 años, mientras que $67 \%$ declaraba no haber hablado de política con personas que no fueran de su familia en los últimos siete días; incluso $44 \%$ afirmó nunca hablar de política.

Este escenario de una juventud mexicana apática en materia política es frecuente en la literatura nacional (Flores, 2000; Monsiváis, 2000) y coincide con otras preocupaciones y hallazgos verificados en países como Gran Bretaña y Estados Unidos. En este sentido, Buckingham (2000) realizó una comparación internacional en jóvenes de estos países y observó que desconfiaban de los actores políticos, observando que ello se traducía en actitudes de cinismo político, apatía, ignorancia y falta de participación. En México, en un concurso de dibujo realizado por la Secretaría de la Función Pública en 2007, se observó que muchos niños describían a un político como una persona que detenta el poder en beneficio propio (Roldán, 2007).

Orozco (2001) menciona que la cultura actual tiene mucho que ver con la televisión, pues los acontecimientos del entorno se apoyan en los

3 Una versión preliminar de este trabajo se presentó en el XX Encuentro Nacional AMIC 2008, “Aportes teóricos-metodológicos de la investigación en comunicación a la teoría social".

Esta investigación se deriva del proyecto apoyado por CONACYT-SEP "Televisión y socialización política de niños en el noreste de México" llevado a cabo en el Centro de Investigación en Comunicación e Información (CINCO), del Tecnológico de Monterrey y dirigido por el doctor José Carlos Lozano y liderado por Enrique Huerta Wong. 
elementos que este medio de distracción, información y entretenimiento aporta, además de que se comporta como un referente común y un foro en el cual las audiencias negocian eventos sociales y asuntos de interés, como lo mencionaban Newcomb e Iris (en Orozco, 2001).

La televisión es el medio que más utilizan los ciudadanos para informarse de lo que ocurre en la política. Así lo ponen de manifiesto los datos de la Encuesta de Cultura Política ENCUP (2001), que señalan que la televisión ha ocupado entre 62 y $74 \%$ de las preferencias reportadas acerca del medio para informarse de política a nivel nacional.

La cultura política de las personas tiene sus orígenes en los procesos de socialización que viven durante su infancia, adolescencia y juventud en interacción con diversos agentes como la familia, los amigos, la escuela y los medios de comunicación, sobre todo la televisión, algo que no tiene por qué ser diferente en los mexicanos. Cullingford (1992) señala que los niños, aunque no tuvieran interés en la política, no podían evitar aprender sobre ella durante su formación. Esta condición se incrementa en tiempos de elecciones, momento en que los medios de comunicación y prioritariamente la televisión aportan referentes del tema político y que, como plantea la teoría de la agenda-setting, pueden formar parte de sus agendas privadas.

Segovia (2001) analizó la politización del niño mexicano aplicando 3,584 encuestas en Jalisco, Monterrey, Oaxaca y escuelas rurales del Estado de México, propiciando un panorama teórico y empírico sobre las concepciones y valores de los niños de ese tiempo. Contrario a los casos internacionales, en este estudio no se encontraron actitudes apáticas ni cínicas, pero el estudio no hace referencia al factor de la televisión. En otro estudio más reciente Tapia (2003), al integrar en su observación a los medios de comunicación como reforzadores de las representaciones sociales y políticas, encontró que los niños que leen periódico y ven la televisión tienen mayor información política.

Por su parte, Ibarra (2003) analizó la oferta televisiva a partir de la programación infantil y entrevistó a padres, maestros y niños, a fin de identificar los distintos factores que se integran en la construcción y gestación de la cultura política en los niños de Guadalajara. El autor concluyó que la televisión es uno de los medios de mayor uso y convivencia con los niños, más allá de un simple refuerzo. En este mismo año 
López Gómez (2003) hizo un estudio comparativo entre el fenómeno en un estado del norte y otro del sur, analizando los procesos de socialización política en los niños de Juchitán (Oaxaca) y Monterrey (Nuevo León). Observó cómo los niños de Juchitán estaban más envueltos en las dinámicas políticas de su entorno, ya que sus padres se hallaban más involucrados en la esfera política y lo transmitían a sus hijos, no sucediendo lo mismo con los niños de Monterrey. El estudio dejó pautas importantes para la observación más a fondo en el caso de esta última ciudad, sobre todo en relación con la apatía política que este estado del norte reportaba frente a uno del sur.

Desde la perspectiva de la psicología política, Nateras (1995) plantea que, dentro del estudio de la socialización política de los niños, debe entenderse que hay estructuras de intercambio y de formación a partir de las cuales las sociedades humanas se reproducen. Además, al igual que Flores (2000), menciona que las representaciones sobre el ciudadano y la ciudadanía dependen de la socialización, el nivel de información, los medios de comunicación y las percepciones sobre actores y órganos de representación. En este sentido, la televisión y las mediaciones pueden ser elementos estructuradores o de agencia para la sociedad, y desempeñan un papel primordial si queremos entender la socialización política de los niños, que, como planteaba Cullingford (1992), no está lejano de las dinámicas y desarrollo de los mismos.

La campaña presidencial de 2006 se apoyó con fuerza en la propaganda televisiva. El IFE (2006) reportó un total de 40,305 promocionales en televisión para candidatos a presidente, del 19 de enero al 28 de junio, con un total de 904,667 segundos de tiempo en televisión, cuyas emisiones aumentaron a medida que se acercaba el 2 de julio. Por otro lado, el tiempo dedicado a partidos políticos o coaliciones en los noticiarios se fue incrementando mensualmente de 176 horas en abril, a 406 en junio. Es posible que, siguiendo la propuesta de Kiousis, McDevitt y $\mathrm{Wu}$ (2005) esta presencia en televisión posibilitaría que el tema político entrara en la agenda de los niños, sobre todo por la discusión en el hogar sobre el tema político.

En el contexto latinoamericano, García Canclini (2004) plantea una sociedad cada vez más despegada de la memoria, cuyas consecuencias tienden a orientarse a la racionalidad individual, coincidente 
con lo que observa Monsiváis (2000) en un estudio sobre la juventud de Baja California.

Estos resultados preocupan por sus proyecciones apáticas, cínicas, aisladas, producto de la desconfianza y que se pueden materializar en acciones como la falta de participación e integración y en algunos casos hasta migración. Muchas de estas actitudes antisociales tienden a gestarse durante la infancia, y el referente común, la televisión, pone en juego diversas implicaciones importantes para su observación.

En la construcción de una cultura política democrática, la socialización política es fundamental para una consolidación de conocimientos, valores y actitudes que estén presentes dentro de las dinámicas sociales con miras a la participación, la solidaridad y el bien común. El análisis de este proceso y del papel que juega un medio de comunicación común es importante si queremos entender y tener fundamentos para activar aquellos aparatos que supuestamente contribuyen al fortalecimiento de una cultura política democrática.

Ante un escenario mexicano de generaciones poco interesadas en los asuntos políticos, y para las que cada vez es más importante el papel de las mediaciones, es preocupación de esta investigación observar cómo se produce este fenómeno en tiempo de elecciones. Así, el presente estudio tiene por objetivo visualizar el papel de la televisión global y los noticiarios en la formación de percepciones sobre la política de niños regiomontanos de primaria, tomando en cuenta dos mediaciones en particular: la familia y el tipo de escuela. Se hará análisis de la recepción a partir de lo que los niños manifiestan y reportan sobre la información que obtienen de la televisión y se buscará conocer más sobre las mediaciones familiares y socioeconómicas que actúan en su proceso de socialización.

En el interior de los medios y en especial de la televisión, existen muchos intereses económicos y de poder que condicionan los contenidos de los mensajes mediáticos y se rigen, cada vez más, por las reglas del mercado y por una comunicación entendida como mercancía (Esteinou, 2003). Este panorama comercial en la televisión, así como el reducido espectro de acción por parte de las legislaciones, genera que los medios de comunicación se mantengan alejados a los principios de una cultura política democrática durante las campañas políticas. Incluso 
que sean útiles a partidos políticos que hacen uso de este espacio para vender una imagen e informar de sus ejes de gobierno.

Jensen (1992), en su estudio sobre "significados múltiples de la política televisada", halló que los procesos políticos suelen conducirse cada vez más a partir de los medios masivos, y que la participación popular depende en mayor medida de una alfabetización funcional en la que se incluyen las habilidades de comprensión y crítica.

Robles (2003) estudió la percepción que los niños de diferentes clases tienen respecto otros estratos sociales representados en la televisión, tomando en cuenta que esta funciona como un engranaje de instancias sociales que acercan a los niños a conocer el mundo social dentro de su desarrollo.

La situación económica y la formación de los padres es una mediación que se involucra en el proceso de socialización política de la audiencia infantil. En este artículo se pretende conocer cómo influyen estas características en el consumo televisivo y cómo van formando parte del statu quo de capital cultural, si es que los patrones de consumo y reproducción se mantienen. También se pretende saber si esto se debe a un déficit cultural que se asocia a una brecha educativa que debería atenderse, que podría decirse genera una interacción cíclica del contenido de los medios y sus audiencias.

La sociología de la educación ha demostrado que los estudiantes de diferentes clases sociales poseen diferentes orientaciones escolares y sistemáticamente se refuerza y genera la legitimación de las formas de conocimiento y capital cultural, que son evaluadas como generación de grupos desaventajados (Buckingham, 2003).

\section{LA RECEPCIÓN COMO ACTO POLÍTICO}

Una de las preocupaciones de este estudio tiene que ver con el proceso de recepción dentro de la socialización política de los niños, que será parte de su formación de concepciones y percepciones sobre la política. Mouffe (1999), en su diferenciación entre la política y lo político señala que la primera se refiere a los mecanismos y formas en que se establece el orden y es organizada la existencia humana que por naturaleza es conflictiva. Por otro lado, lo político es la cualidad de las relaciones 
entre las existencias humanas expresada por la diversidad de relaciones humanas en su coexistencia.

Tomando en cuenta estas diferencias, es pertinente entender que los contenidos televisivos en tiempos electorales dan una muestra de la política, así como de los roles activos de lo que establece el orden y organiza: actores, instituciones, partidos, entre otras. Y, por otro lado, el proceso de recepción genera un entorno de lo político cuando interactúan, se comunican y se ponen en negociación modelos de interpretación de un referente en común, lo televisado de la política.

La recepción es el proceso anterior y posterior al momento de estar expuesto a la televisión (Orozco, 1992), no sólo es recibir, es una interacción siempre mediada por diversas fuentes y contextos materiales, cognitivos y emocionales presentes en este proceso complejo, generando distintas apropiaciones. Es un consumo cultural a partir del cual, como señala Calles (1999), las identidades se forjan y se van delineando hacia formas de acción.

Jensen (1992) señala que el estudio de la recepción debe examinarse a fin de valorar concretamente el poder de los medios frente a las audiencias y conocer cómo se forma a las audiencias-ciudadanos.

Desde los estudios culturales se entiende que el proceso de recepción se inicia en la decodificación de un mensaje por parte de la audiencia. Este mensaje, como lo señaló Fiske (1987), consiste en la posibilidad de interpretaciones que se pueden tener de un solo texto, es decir, de la diferente decodificación por diferentes audiencias según las convenciones culturales de sus antecedentes.

La interpretación de los mensajes depende de las mediaciones implicadas en el proceso de recepción. Las mediaciones en sí mismas cumplen un papel político de orientación en la recepción. Como señala Jensen (1992), la polisemia es entonces un concepto político y la recepción en sí misma también puede considerarse como un acto político, ya que como plantea Orozco (1992) es en la recepción donde puede darse la manipulación o emancipación de las audiencias.

La recepción, dice Lozano (1991) basándose en los postulados de Martín Barbero, no es pasiva sino que atraviesa una serie de mediaciones que articulan contenidos con la rutina en el contexto familiar, comunitario y nacional. Martín Barbero (en Lozano, 1991) señala que 
los tres lugares claves de la mediación son la cotidianidad familiar, la temporalidad social y la competencia cultural.

Una campaña política "cristaliza" las actitudes y predisposiciones en lo político de niños y adolescentes, en la medida en que se exponen a los contenidos políticos de los medios y se enriquece con la conversación de los padres (Sears y Valentino, 1998).

Las investigaciones desde los estudios culturales (Ang, 1991; Fiske, 1987; Hall, 2001; Morley, 1992) han centrado su atención en la forma en que las audiencias se apropian de los contenidos televisivos y del tipo de lecturas que hacen ante la información que presentan, si hay un control hegemonizador de los medios y si estas audiencias responden de alguna manera. El tópico de la hegemonía remite a las negociaciones del poder y por tanto a un tema político en la audiencia. Sin embargo, como se discutió anteriormente, lo político no está necesariamente enfocado a los contenidos de la política y su apropiación en relación con esta.

Por otro lado, los estudios de comunicación política han tratado de entender la relación y el papel que juegan los medios con respecto a la relación gobernantes y gobernados, los usos propagandísticos que se dan, los usos ciudadanos y los efectos e impactos orientados, sobre todo a la participación de la audiencia en el marco de la democracia (Chaffe, 1997; Jensen, 1992; Liebes y Katz 1989; McCombs, 2006; Sears y Valentino, 1998). Sin embargo son pocos los trabajos que analizan el papel que juega la televisión en el proceso de socialización política de los niños en este entorno de comunicación política y recepción (Kiousis, 2005).

De acuerdo con Gunter y McAleer (1997), el conocimiento sobre la política se relaciona con el uso de los medios masivos y, especialmente, con el consumo de noticias y de temas actuales. Esta influencia rebasa el plano de los medios tradicionales.

Según Meirick (2004) los teóricos típicamente señalan que un electorado bien informado es esencial para el funcionamiento de la democracia. Además, argumentan que las personas con bajo conocimiento suelen carecer de voz y opinión en un proceso democrático. No obstante, también existen teorías que dicen que no necesariamente se debe tener información para saber lo que se quiere y lo que está ligado a sus intereses y valores, lo político, tal como lo ha investigado Popkin (1991, en Meirick, 2004). 
La recepción tiene implicaciones políticas; es un proceso que involucra la conformación de actitudes, conocimientos y percepciones acerca de la política, posicionándose sobre la estructura de esta. Se podría hablar, por tanto, de un empoderamiento gracias a la información que proporciona la información televisada o, como plantea Buckingham (2000), de percepciones negativas que conducen a actitudes como cinismo, apatía, desconfianza.

\section{LA BRECHA EDUCATIVA, UNA MEDIACIÓN LATENTE}

Bourdieu (2000) señala que las relaciones entre el sistema de enseñanza y la estructura de las clases sociales están relacionadas con el sistema escolar, expresando estrategias diferentes de la inversión cultural no garantizada por la institución escolar. Señala una fuerte relación entre el grado de estudios y el nivel socioeconómico. Aun cuando la preparación educativa en el plano discursivo y utópico está al alcance de todos, en el plano operacional se comporta de otra manera.

El nivel socioeconómico ha sido una variable que evidencia diferencias en consumo y apropiación de los mensajes comunicacionales, remarcando la brecha en poder y cultura (Lozano, 2000). Esta estratificación, como señalaban Croteau y Hoynes (2003), actúa como mediadora central en el proceso interpretativo, ya que provee estratégicamente recursos para decodificar los medios a partir del utillaje cultural y recursos discursivos, pues este entorno genera el marco por el cual vemos o no las cosas, lo visible o invisible desde las herramientas al alcance. Además, estos autores señalan que tal utillaje es usado para construir significados en patrones específicos. Esta condición estructural específica, condiciona la posibilidad del agente activo de esta relativa autonomía que plantea Lull (1997).

Giddens (en Orozco, 1996:36) enfatiza que la actividad social se estructura por reglas y entendidos cotidianos "que construyen y regulan patrones de conducta social y reflejan valores culturales legitimados, concretados y extendidos por historias específicas de acción social". Es decir, plantea que hay un entorno de dinámicas que por sus prácticas se han "legalizado", y a esto se puede agregar que son heredadas y se alfabetizan o no en el camino. 
Guillermo Delahanty (1995) menciona que es durante el desarrollo del niño cuando se asimilan ciertas nociones sociales y políticas procedentes de la familia, utilizadas para construir los esquemas mentales de los que se servirán para comprender las relaciones de poder.

El capital cultural aportado por los padres, así como por las instituciones educativas, es un condicionante que está presente en la interpretación de las audiencias infantiles.

La mediación familiar en el contexto de una recepción global de la televisión ha sido estudiada por varios autores (Charles 1992, Orozco, 2001, Renero, 1992, Sánchez, 1992), destacando cierto consenso sobre su importancia para comprender la complejidad de las interacciones en el proceso de recepción y tener mayores fundamentos y propuestas para los usos o abusos del medio masivo, en la conciencia de su función como articulador de interpretaciones y percepciones.

Como señala González (2001), existimos en un medio de intrincado y dinámico flujo de discursos sociales, donde algunos de ellos provienen de organizaciones proteccionistas, con la intención de definir, regular y concentrar el significado común de nuestras necesidades, identidades y valores "considerarlos como dignos de obtener y preservar en la sociedad". El estudio de la recepción permite acercarnos a la forma en que el flujo de discursos sociales y políticos se va configurando en la percepción de los receptores.

Como mencionaban Croteau y Hoyness (2003), las diferentes posiciones sociales proporcionan distintos recursos discursivos, lo que hace que algunas cosas sean más perceptibles que otras, en función de las herramientas culturales disponibles, y que es un hecho que estas no están al alcance de todos.

\section{MÉTODO}

\section{Procedimiento}

Se efectuaron entrevistas focalizadas y encuestas en tres ciudades del noreste mexicano: Monterrey (Nuevo León), Saltillo (Coahuila) y Ciudad Victoria (Tamaulipas). La presente investigación se realizó a partir de los datos de Monterrey aprovechando la triangulación de técnicas de investigación cualitativas y cuantitativas para dar mayor dimensión al estudio. 
La información cuantitativa ayudará a vislumbrar el panorama sobre todo en función del tipo de escuelas, dando un escenario sobre el capital cultural que tienen los niños, y acercarnos a la situación de la brecha educativa, su relación con la recepción televisiva y de contenido político.

Aunque aquí se reporta un diseño metodológico en función del sujeto de estudio, el estudio cuantitativo se apoyó en lo explorado por el estudio cualitativo; no se deriva de sus resultados sólo de sus observaciones para el diseño del cuestionario. Por esta razón, los resultados serán expuestos de lo general a lo particular con la intención de problematizar, a partir de lo planteado en el marco teórico, desde el análisis cuantitativo de la brecha educativa y de ahí ir a la discusión cualitativa, dado que se analiza desde la perspectiva de los estudios culturales.

A partir de los datos reportados en estudios empíricos sobre cultura política y de las consideraciones teóricas encontradas en la revisión de la literatura, nos interesó conocer en el estudio cuantitativo el perfil demográfico en cada tipo de escuela, el tipo de comunicación interpersonal y tipo de consumo televisivo.

\section{Diseño de la investigación cuantitativa}

Con el objetivo de obtener información general sobre el entendimiento de la construcción de la cultura política de los niños de Monterrey desde las percepciones generadas por el proceso de recepción y, así conocer el papel que juega la televisión, se realizó un estudio mediante cuestionario en junio de 2006. La unidad de selección fueron escuelas primarias, y los niños cursantes de sexto año de primaria la unidad de observación; participaron un total de 588 sujetos.

La edad de los niños encuestados variaba entre los 11 y los 15 años $(M=11.86, D E=0.59)$. El estudio se aplicó a los niños de $6^{\circ}$ año, al igual que se hizo en el estudio de Cullingford (1992), al considerarse que en seis años estos niños tendrán edad de votar en la siguiente elección presidencial. Además, de los 588 niños $49 \%$ eran niños y $46 \%$ eran niñas.

A fin de generar un mejor instrumento, se recurrió primero a la técnica cualitativa para acercarse al contexto de los niños y posteriormente generar el cuestionario tomando en cuenta lo observado y reportado.

Las encuestas se aplicaron presencialmente sobre una muestra de 32 escuelas $(N=588)$ representativas del universo de estudio (474 escue- 
las). A partir del listado oficial de la Secretaría de Educación Pública (SEP, 2006), se obtuvo una muestra probabilística por conglomerados, en la proporción tanto de las escuelas públicas como de privadas, a fin de poder realizar comparaciones entre las escuelas, 22 públicas $(N=454)$ y 11 privadas $(N=134)$.

En caso de que hubiera más de un aula se eligió al azar una y se aplicó el cuestionario a todos los niños del salón. Todos los datos se capturaron en excel y se procesaron en SPSS v.15.0.

El cuestionario constaba de 72 items, de los cuales para este estudio se tomaron únicamente 29 , sobre comunicación interpersonal (6 items), sobre el conocimiento de los actores políticos (7 items), las percepciones, actitudes y opiniones sobre la política (9 items), sobre el tipo de consumo televisivo (5 items) y de educación de los padres (2 items). Se realizó una prueba piloto en Monterrey con niños de una escuela privada y otra pública para asegurar la legibilidad y claridad del cuestionario.

Uno de los objetivos de este estudio consistió en conocer cómo se desarrollan las percepciones de la política televisada de los niños de acuerdo al tipo de escuela, a las redes de comunicación interpersonal y al tipo de consumo televisivo para acercarnos más al fenómeno de sus percepciones. $\mathrm{Al}$ respecto se les preguntó:

Tamaño de las redes de comunicación interpersonal política: “¿Qué tanto platicas de política con tu papá?”, “¿Qué tanto platicas de política con tu mamá?”, “¿Qué tanto platicas de política con tu abuelito o abuelita?", "Qué tanto platicas de política con tus compañeros de escuela?", “¿Qué tanto platicas de política con tus primos y hermanos?”, “QQué tanto platicas de política con tus maestros?".

Nivel educativo de los padres: las medias para cada tipo de escuelas.

Conocimiento de los actores políticos: suma de respuestas correctas respecto a la identificación de Vicente Fox Quesada (presidente), Andrés Manuel López Obrador (candidato del PRD), Roberto Madrazo Pintado (candidato del PRI), Felipe Calderón Hinojosa (candidato del PAN), Roberto Campa Cifrián (candidato de Nueva Alianza) y Patricia Mercado (candidata de Alternativa).

Exposición a la televisión: horas vistas de lunes a viernes y horas vistas el fin de semana. 
Exposición a programas de noticias: “¿Qué tanto ves noticias nacionales?”, ¿Qué tanto ves noticias locales?”.

Percepciones de la política y los políticos: “¿Consideran a la política mala?”, “¿Consideran políticos corruptos?”.

Actitudes hacia política y políticos: "Consideran que votar es importante", "Me gustaría ser diputado", "Me gustaría ser gobernador", "Me gustaría formar un sindicato", "Me gustaría unirme para mejorar el país".

\section{Diseño de la investigación cualitativa}

El trabajo de campo fue realizado en marzo de 2006, y en él que se llevaron a cabo entrevistas focalizadas (Lindolf, 1995), realizadas a partir de una guía de preguntas a un pequeño grupo de personas. Un estudio reciente (López Gómez, 2003) sugiere que el mejor modo de estudiar este particular fenómeno con niños es reunir a pequeños grupos, de 3 ó 4 miembros, para disminuir la intimidación que los niños puedan sentir hacia el investigador y el tema, lo que se confirmó en la realización de las entrevistas: los niños se sentían más en confianza, sobre todo si no eran abordados de inmediato con las preguntas y se tenía un primer acercamiento, para lo cual se recurrió a preguntar sobre la actividad que estaban realizando en el salón de clases, estableciendo rapport. Como señalan Taylor y Bogdan (1987), la intención es entender el fenómeno social desde la perspectiva que tiene el sujeto y acercándonos más a su naturalidad, reconociendo las consecuencias que la presencia del investigador causa.

Cada entrevista duró alrededor de 30 a 40 minutos y todas se transcribieron. La muestra fue por conveniencia y contraste, ya que interesaba diferenciar los procesos de socialización política entre una escuela privada y una pública mediante una visión cualitativa a fin de profundizar en la experiencia subjetiva de los procesos de apropiación del contenido televisivo.

La prueba piloto fue aplicada en la primaria pública para revisar el guión de entrevista a fin de asegurar claridad y recoger la información que se deseaba. Se hicieron algunas correcciones y finalmente se aplicó en una escuela pública y en una privada de Monterrey. 
Se entrevistó a 30 niños de 10 a 12 años (15 niñas y 15 niños) de cada escuela de Monterrey, Nuevo León, en entrevistas grupales de tres. El diseño de la guía de entrevista se realizó a partir de la revisión de la literatura y bajo la coordinación del doctor Enrique Huerta. En estas se llegó a la saturación, es decir los niños repetían la misma información en la mayoría de los casos, no siendo necesario la aplicación de más entrevistas, que fueron grabadas en audio y transcritas para su interpretación.

El análisis se concentró en los argumentos, ejemplos, recuerdos, percepciones y discusiones de los niños con la finalidad de profundizar en la experiencia subjetiva de la apropiación de contenidos políticos televisados.

Este estudio complementa la información obtenida de encuestas y entrevistas entendiendo sus potencialidades y limitaciones, a fin de contrastar, triangular y tener dos dimensiones del fenómeno en Monterrey a partir de los objetivos del presente estudio en la recepción televisiva.

\section{RESULTADOS}

Bourdieu (2000) establece que hay una relación entre el capital heredado y el capital escolar, y menciona que también existe un efecto de conversión desigual del capital cultural heredado, ya que lo que les exigen se reduce sólo a aquello que los padres lograron conseguir como máximo en su formación.

En el presente estudio se encontró que en la escuela privada de Monterrey más de la mitad de los padres $(63 \%)$ y las madres $(59 \%)$ estudiaban la universidad. En cambio, en la escuela pública el mayor porcentaje de escolaridad del padre (43\%) y de la madre (41\%) se ubica en la secundaria. Esto da cuenta de una brecha en la formación de los padres, que, de acuerdo a Bourdieu (idem) son unos de los más importantes portadores del capital cultural y reproductor de los conocimientos, valoraciones y actitudes.

Se preguntó a los niños con quiénes platicaban sobre política (tabla 1) y la mayoría reportó hablar muy poco del tema. Aun así, apoyado en una Prueba t de Student para muestras independientes, los niños de la escuela privada señalaban tener más plática con el padre y compañeros que los niños de la escuela pública. Con maestros, abuelos, hermanos 
no se encontró diferencia entre los grupos. La plática con la madre se comporta igual en ambas escuelas, y es más alta que la media de plática con abuelos y hermanos; en estos tres agentes no hay una diferencia significativa entre ambas escuelas (véase tabla 1).

\section{TABLA 1}

MEDIAS DE PLÁTICA CON LOS DIVERSOS ACTORES SOCIALES, EN FUNCIÓN DEL TIPO DE ESCUELA (T DE STUDENT)

\begin{tabular}{|c|c|c|c|c|c|}
\hline \multirow{2}{*}{$\begin{array}{l}\text { Nivel plática de } \\
\text { política con }\end{array}$} & \multirow{2}{*}{$\begin{array}{c}\text { Media } \\
\text { total }\end{array}$} & \multicolumn{2}{|c|}{ Tipo de escuela } & \multirow[t]{2}{*}{$\mathrm{t}(\mathrm{gl})$} & \multirow[t]{2}{*}{$\mathrm{p}$} \\
\hline & & Pública & Privada & & \\
\hline \multirow[t]{2}{*}{ Padre } & 1.60 & 1.52 & 1.84 & -2.723 & \\
\hline & $(1.27)$ & $(1.30)$ & $(1.14)$ & $(245)$ & .007 \\
\hline $\mathrm{N}$ & 570 & 437 & 133 & & \\
\hline \multirow{2}{*}{ Madre } & 1.64 & 1.60 & 1.79 & -1.571 & \\
\hline & $(1.22)$ & $(1.25)$ & $(1.12)$ & $(239)$ & .097 \\
\hline $\mathrm{N}$ & 575 & 442 & 133 & & \\
\hline \multirow[t]{2}{*}{ Abuelo } & 1.09 & 1.06 & 1.20 & 1.083 & \\
\hline & $(1.2)$ & $(1.23)$ & $(1.27)$ & $(203)$ & .280 \\
\hline $\mathrm{N}$ & 566 & 435 & 131 & & \\
\hline \multirow[t]{2}{*}{ Compañeros } & 1.12 & .99 & 1.55 & -4.525 & \\
\hline & $(1.27)$ & $(1.22)$ & $(1.32)$ & (569) & .000 \\
\hline $\mathrm{N}$ & 571 & 438 & 133 & & \\
\hline \multirow[t]{2}{*}{ Hermanos } & 1.04 & 1.03 & 1.08 & -.352 & \\
\hline & $(1.29)$ & $(1.28)$ & $(1.30)$ & $(215)$ & .725 \\
\hline $\mathrm{N}$ & 562 & 430 & 132 & & \\
\hline \multirow[t]{2}{*}{ Maestros } & 1.34 & 1.31 & 1.45 & -1.291 & \\
\hline & $(1.16)$ & $(1.13)$ & $(1.26)$ & $(566)$ & .197 \\
\hline $\mathrm{N}$ & 568 & 436 & 132 & & \\
\hline Índice de red de & 1.29 & 1.23 & 1.48 & -3.466 & \\
\hline comunicación política & $(0.75)$ & $(0.72)$ & $(0.76)$ & $(231)$ & .001 \\
\hline $\mathrm{N}$ & 588 & 408 & 131 & & \\
\hline
\end{tabular}

Nota 1: El rango teórico de variación de las variables dependientes fue de 0 (nunca) a 3 (mucho).

Nota 2: Los valores entre paréntesis representan la desviación estándar de las medias. 
Al realizar una nueva prueba $t$ en función del índice de la red de plática política que tienen en cada escuela, mediante la suma de todas las variables, se observó que los niños de la escuela privada reportan tener más plática sobre política $(M=1.48, D E=0.78), t(231)=$ $-3,466, p<.001$, que los de la escuela pública $(M=1.23, D E=0.72)$, $t(231)=-3,466, p<.001$.

Para acercarse a las nociones que los niños tienen de la política, sobre todo en tiempo electoral, se les pidió que ubicaran a los candidatos a la presidencia así como a otros políticos con cargos de responsabilidad como Vicente Fox, presidente de la República y Natividad González, gobernador de Monterrey (véase tabla 2).

\section{TABLA 2}

DIFERENCIAS DE CONOCIMIENTO DE LOS DISTINTOS ACTORES POLÍTICOS, EN FUNCIÓN DEL TIPO DE ESCUELA (PORCENTAJE COLUMNA)

\begin{tabular}{cccccc} 
Políticos & \multicolumn{2}{c}{ Tipo de escuela } & Total & $\chi^{2}(1)$ & $\mathrm{p}$ \\
& Pública & Privada & & & \\
\hline Vicente Fox & 77 & 85 & 79 & 3.055 & .080 \\
$\mathrm{~N}$ & $(433)$ & $(132)$ & $(565)$ & & \\
\hline López Obrador & 67 & 697 & 67 & 0.336 & .562 \\
$\mathrm{~N}$ & $(421)$ & $(131)$ & $(552)$ & & \\
\hline Roberto Madrazo & 54 & 66 & 57 & 5.817 & .016 \\
$\mathrm{~N}$ & $(226)$ & $(87)$ & $(313)$ & & \\
\hline Felipe Calderón & 52 & 65 & 55 & 7.357 & .008 \\
$\mathrm{~N}$ & $(214)$ & $(85)$ & $(299)$ & & \\
\hline Natividad González & 72 & 81 & 74 & 4.949 & .030 \\
$\mathrm{~N}$ & $(305)$ & $(106)$ & $(411)$ & & \\
\hline Roberto Campa & 41 & 46 & 42 & 1.025 & .359 \\
$\mathrm{~N}$ & $(69)$ & $(60)$ & $(229)$ & & \\
\hline Patricia Mercado & 46 & 47 & 46 & .089 & .840 \\
$\mathrm{~N}$ & $(188)$ & $(62)$ & $(250)$ & & \\
\hline
\end{tabular}

Nota: A efectos de elaborar una tabla final agrupada con los datos de cada actor político, sólo se han reportado los porcentajes de niños que contestaron correctamente. 
Al realizar una nueva prueba $t$ de muestras independientes, esta vez para detectar las diferencias entre las medias de las escuelas privada y pública, se observó que los niños de privada, en promedio, de siete actores políticos identifican correctamente a 4.56 de ellos; en la escuela pública identifican correctamente a un promedio de $3.93, t(567)=$ $-2.764, p<.001$. Los niños de ambas escuelas identifican en primer lugar a la figura del presidente (79.5\%) seguido por la de su gobernador (74.1\%). Estos datos confirman en pequeña medida lo que la prueba de conocimiento ENLACE mostraba: una ventaja en los conocimientos de las escuelas privadas sobre las públicas.

Los niños de la escuela privada identificaron más que los de la pública a Vicente Fox, al gobernador Natividad González, a Roberto Madrazo y a Felipe Calderón, tenían más conocimiento de ellos, pero para el resto de los actores el conocimiento fue el mismo.

El intercambio de información política a través de la conversación y la exposición a la televisión son experiencias que, como lo han mencionado diversos autores (Bukingham, 2003; Ibarra, 2003 y Tapia 2003), permiten a los niños acercarse al tema de la política, ya que les proporciona sus primeras nociones sobre los actores, instituciones y los procesos.

Un referente común para ambos tipos de escuela es la televisión. Los niños en México pasan largos periodos frente a esta, aproximadamente 4 horas (Jara, 2007). En este estudio (tabla 3) se reportó un tiempo global promedio de 4.3 horas de los niños en ambas escuelas. Los niños de escuela privada ven menos televisión, entre semana $(M=3.6, D E=1.75)$, que los de la escuela pública $(M=4.11, D E=3.64), t(541)=2.154$, $p<0.05$. Sin embargo, el fin de semana no hay diferencia en el consumo de horas, ambos tienen un promedio de consumo igual a 3.6 horas.

Los niños estaban mayormente expuestos a la información política con el tratamiento de los noticiarios de la región. Noticias locales eran consumidas más por niños de la escuela pública (27\%) que por los de la privada $(15.7 \%)$.

Opiniones y valoraciones de "la política" desde "lo político"

Se codificaron los cuatro factores a los que se asignó un nombre que explicara el conjunto (tabla 4). El primer componente denominado "opinión sobre la participación política" estaba compuesto por las 
variables: qué tan de acuerdo están con ser "diputado", "gobernador" y "formar un sindicato". El segundo factor denominado "valora-

\section{TABLA 3}

MEDIAS DE HORAS VISTAS DE TELEVISIÓN, EN FUNCIÓN DEL TIPO DE ESCUELA (T DE STUDENT)

\begin{tabular}{|c|c|c|c|c|}
\hline \multirow[t]{2}{*}{ Horas que ve televisión } & \multicolumn{2}{|c|}{ Tipo de escuela } & \multirow[t]{2}{*}{$\mathrm{t}(\mathrm{g} . \mathrm{l})}$. & \multirow[t]{2}{*}{$\mathrm{p}$} \\
\hline & Pública & Privada & & \\
\hline Entre semana & $4.11(3.64)$ & $3.60(1.75)$ & 2.154 & .032 \\
\hline $\mathrm{N}$ & 415 & 128 & (541) & \\
\hline El fin de semana & $3.60(2.89)$ & 3.57 (1.90) & 0.160 & .873 \\
\hline $\mathrm{N}$ & 406 & 129 & $(187)$ & \\
\hline
\end{tabular}

\section{TABLA 4}

COMPOSICIÓN Y CONSISTENCIA INTERNA (ALFAS DE CRONBACH) DE LAS DIFERENTES OPINIONES, VALORACIONES Y CONSIDERACIONES POLÍTICAS

Opiniones sobre la política

$\alpha$

Opinión sobre la participación política

Estoy de acuerdo con ser diputado

Formar sindicato

Ser gobernador

Valoración de la comunicación interpersonal

La comunicación con mis padres es buena

Mis maestros me toman en cuenta

Los maestros deben escuchar a sus alumnos respecto al mejoramiento de la escuela

Es más fácil que yo me disculpe a que me pelee

Valoración de la participación electoral

Votar en esta elección es importante

Votar es importante

Valoración de la política

Los políticos son corruptos

La política es mala 
ción de la comunicación interpersonal" estaba compuesto por "la comunicación con mis padres es buena", "mis maestros me toman en cuenta", "los maestros deben escuchar a sus alumnos respecto al mejoramiento de la escuela" y "es más fácil que yo me disculpe a que me pelee". El tercer factor nombrado "valoración de la participación electoral" estaba compuesto por: "votar en esta elección es importante" y "votar es importante". Y el cuarto factor denominado "valoración de la política" se compuso por los items: "los políticos no son corruptos" y "la política no es mala", siguiendo procedimientos propuestos por Igartua (2006).

En los niños de la escuela pública la opinión sobre participación política se relaciona significativamente tanto con el consumo de noticias locales $(r=.163, p<.001)$, como con la valoración de la comunicación interpersonal $(r=.250, p<.001)$, pero esta última con mayor fuerza, con menor significancia con el consumo de noticias nacionales $(r=.110$, $p<.010)$. Además la plática política con el padre también es significativa $(r=.185, p<.001)$; sin embargo su educación se relaciona igual pero de forma negativa $(r=-.045, p<.001)$. A mayor educación de los padres los niños parecieran presentar menos interés en la participación política (véase tabla 5).

En el reciente estudio de Flores (2006) se observó que el voto tiene connotaciones que trascienden al mero ámbito de la competencia y expresa un intenso vínculo con el cambio político, comenzando a tomar sentido de utilidad. La valoración de la participación electoral, en los niños de Monterrey, de forma curiosa, no se relaciona con el consumo de noticias nacionales, sino con el consumo de noticias locales $(r=$ $.113, p<.05) \mathrm{y}$ con mucha fuerza con la valoración que tienen de la comunicación interpersonal $(r=.421, p<.001)$, pero no con la plática política con el padre $(r=.038, p=.372)$, y muy poco con la educación de éste $(r=.093, p<.05)$.

Respecto a la valoración que tienen de la política como tal, sólo hay relación con el consumo de noticias locales $(r=.084, p<.05)$, no existiendo relación ni con el consumo de noticias nacionales $(r=.001$, $p=.998)$, ni con la valoración de la comunicación interpersonal $(r=$ $.081, p=.058)$, así como tampoco con la plática con el padre $(r=.028$, $p=510)$, pero sí negativamente con su formación, aunque no de manera significativa $(r=-.091, p=.510)$. 


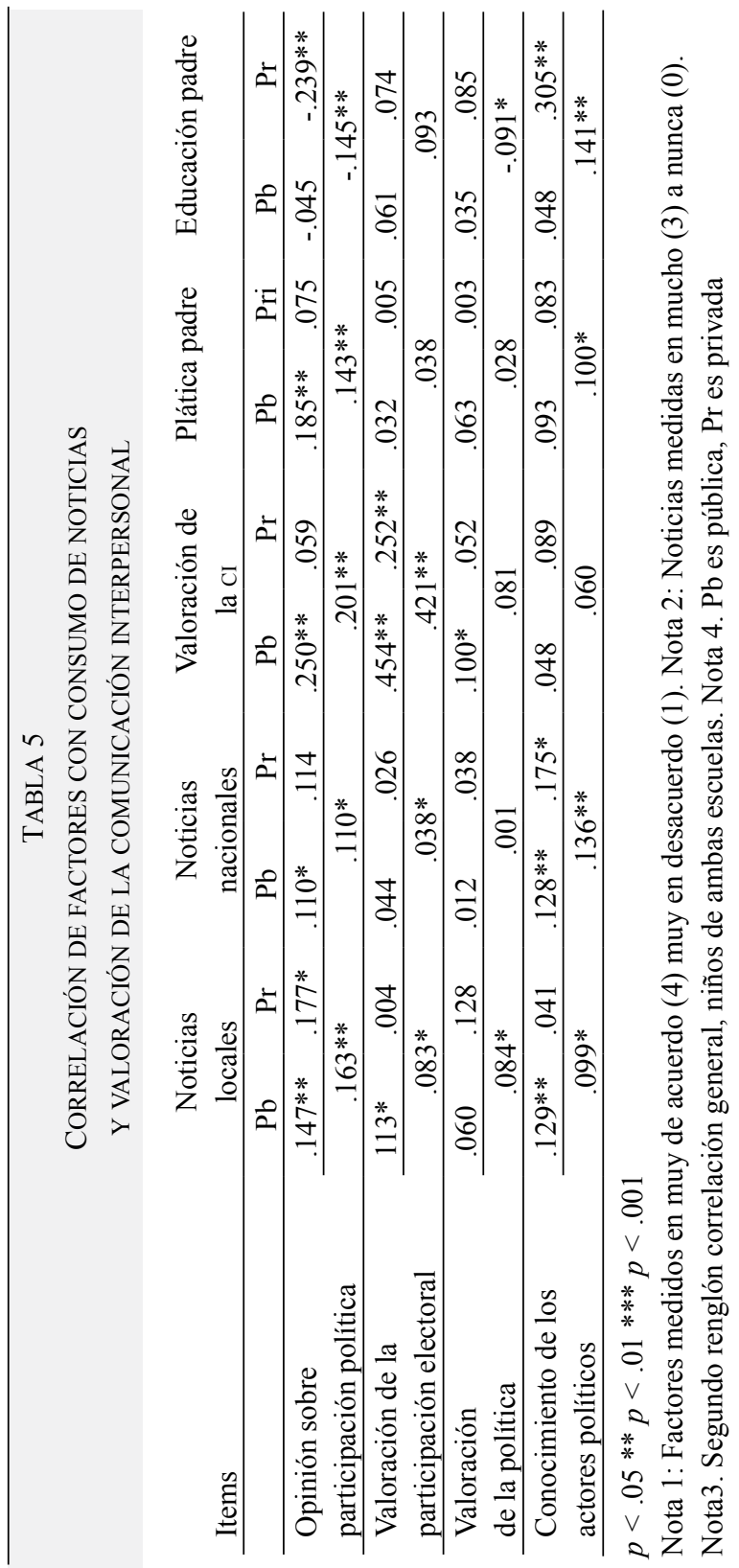


Respecto al conocimiento de los actores políticos se encontró que el consumo de noticias nacionales estaba significativamente relacionada $(r=.136, p<.001)$ y también con el consumo de noticias locales $(r=.099, p<.05)$, pero sin relación con la valoración de la comunicación interpersonal $(r=.060, p=.161)$, pero sí por la plática política con el padre $(r=.100, p<.050)$ y con más significación de acuerdo a la formación de este $(r=.141, p<.001)$.

En términos generales, podemos observar la relación de los niños con sus valoraciones y opiniones en el momento de aprendizaje en el que se encuentran, por lo que valdrá la pena observar estas características para otros grupos de edad y observar que estas relaciones han evolucionado, generando opiniones como Flores (2006) señalaba en su estudio.

En una Prueba $t$ de muestras independientes se encontró que los niños de la escuela privada tienen la misma valoración a la comunicación interpersonal que los de la pública $(M=3.4, D E=0.60)$; sin embargo, en el caso de la valoración de la participación electoral, los niños de la privada tienen más valoración $(M=3.63, D E=0.64)$ que los de la pública $(M=3.44, D E=0.76)$. Por otro lado, la opinión que tienen sobre la participación política se comporta diferente: en la pública es mayor $(M=2.38$, $D E=0.81)$ que en la privada $(M=2.09, D E=0.79)$. Al igual que la valoración de la política, en la escuela pública es mayor $(M=2.63$, $D E=0.79)$ que en la privada $(M=2.38, D E=0.73)$ (tabla 6$)$.

Como muestran los datos cuantitativos, se puede observar una asimilación de la política y lo político distinto tanto en los niños de escuelas públicas como de los niños de escuelas privadas. De forma general, se encuentra una brecha en la preparación de los padres y los conocimientos sobre los actores políticos.

Las actitudes y opiniones en los niños de la escuela pública están fuertemente relacionadas con la valoración que tienen de la comunicación interpersonal. Tanto en lo que reportan los niños de la escuela pública como los de la privada, la valoración de la participación electoral está fuertemente relacionada con la valoración que tienen de la comunicación interpersonal, tomando en cuenta además que la valoración de la participación electoral es más valorada en la escuela privada $(M=3.4$, $D E=0.53$ ). Por otro lado, su opinión sobre la participación política no se comporta igual; sí teniendo relación con la comunicación interperso- 


\section{TABLA 6}

MEDIAS DE FACTORES, EN FUNCIÓN DEL TIPO DE ESCUELA (T DE STUDENT)

\begin{tabular}{lcccc}
\multicolumn{1}{c}{ Medias de factores } & \multicolumn{2}{c}{ Tipo de escuela } & $\mathrm{t}(\mathrm{gl})$ & $\mathrm{p}$ \\
& Pública & Privada & & \\
\hline Valoración de & 3.32 & 3.40 & -1.195 & \\
la comunicación interpersonal & $(.643)$ & $(.527)$ & $(558)$ & .232 \\
$\mathrm{~N}$ & 428 & 132 & & \\
\hline Valoración de & 3.44 & 3.62 & -2.790 & \\
la participación electoral & $(.762)$ & $(.638)$ & $(258)$ & .006 \\
$\mathrm{~N}$ & 441 & 134 & & \\
\hline Opinión sobre & 2.38 & 2.09 & 3.552 & \\
la participación política & $(.812)$ & $(.789)$ & $(546)$ & .000 \\
$\mathrm{~N}$ & 419 & 129 & & \\
\hline Valoración de la política & 2.63 & 2.38 & -3.340 & \\
$\mathrm{~N}$ & $(.786)$ & $(.731)$ & $(565)$ & .001 \\
\hline
\end{tabular}

Escala de 4 (muy de acuerdo) a 1 (muy en desacuerdo).

nal para la escuela pública, pero no para la privada, y recordando que la opinión que tienen los niños de la pública tiende a ser de más acuerdo $(M=2.4, D E=0.81)$. En la escuela pública la valoración de la política está relacionada con la comunicación interpersonal, y además su valoración de aquella es más alta $(M=2.6, D E=0.79)$ que en la privada, que además no está relacionada con ninguna de las variables comparadas. El conocimiento de los actores políticos se comporta de manera similar y está significativamente relacionado con el consumo de noticias locales y un poco menos con noticias nacionales; en la privada, además, se relaciona con el grado de estudios del padre, sin dejar de lado que los niños de esta tuvieron más conocimiento de los actores políticos que los niños de la pública.

Los datos cuantitativos nos permiten comprender la relación de las condiciones sociodemográficas que reportan el panorama de sus relaciones con el tipo de consumo televisivo y de noticias, a fin de acercarnos al papel que juega la televisión dentro de la cultura política de los 
niños y conocer las condiciones como una fotografía aérea, problematizando la polarización en el reparto y acceso a bienes culturales. La manera en que se están socializando a partir de lo que manifiestan hasta el momento en que fueron entrevistados y el papel que ocupa la televisión se analizará desde el estudio cualitativo en el siguiente apartado.

\section{TELEVISIÓN Y POLÍTICA ¿PARA QUÉ?}

Cullingford (1992) señala que a pesar del poco o mucho interés que los niños tengan en la política, no podrán evitar aprender de esta. Además, estas primeras percepciones también adquieren importancia en su entendimiento de la comunicación política.

Los niños de la escuela pública señalaban ver más televisión y se enteraban de la política por los spots de gobernación, o por aquellos en que se promocionaba a los candidatos; incluso señalaban que si pudieran votar por alguno sería porque lo han visto en la televisión ayudando, tal como señala Hiram:

\section{— ¿Y tú José?}

José: "Por Madrazo porque veo que porque sí está viendo en cualquier parte qué necesiten va a ayudarlos, no tanto, pero sí los ayuda".

— ¿Y tú Hiram?

Hiram: "Yo también porque sí lo he visto en la tele, que pasa que va ayudando a varias personas o algo así".

Escuela pública, 12 años.

También los niños de la escuela privada señalaron que se enteraban de los políticos por los spots que aparecían entre su programación.

- ¿Y por ejemplo, cuando lo ven en la tele, en qué tipo de programa ven cosas de política?

Daniela: "En el canal 5".

Lucía: "Bueno, en el canal 5; en los comerciales siempre sale Fox o Felipe Calderón".

Daniela: "O Felipe Calderón o Roberto".

Escuela privada, 12 años. 
En el caso de los niños de la escuela privada, las opiniones suelen estar más orientadas por los comentarios de sus padres, pero en caso de los niños de la pública pareciera que lo están consumiendo tal como el medio se los presenta; si los padres no los orientan ellos tomarán de referente otro recurso y parece que será la televisión, como menciona Cullingford (1992), pues su cualidad visual posibilita mayores recursos para su memoria y conocimientos.

Los niños de la escuela privada mencionaban enterarse de la política sobre todo porque a sus padres les interesaba conocer quién iba a ganar y las implicaciones que tienen para ellos. Esto, desde la teoría de la agenda-setting, se concibe como un efecto de tema experiencial (McCombs, 2006), es decir que intervienen en la vida personal y cotidiana, por lo cual también le dan seguimiento. A su vez, estos comentarios pudieran darles un entendido de la competencia política entre actores en búsqueda de un puesto y ciertos beneficios.

\section{- ¿Y tú?}

Héctor: "Es que si le pregunto a mi papá, pero por qué, y me dice es que es el papá de éste, o es el amigo que tiene un puesto mayor que yo y me puede ir mejor".

Ricardo: "Si llegara a ganar Madrazo, aunque quién sabe, Monterrey es Nati, es del PRI y si llegara a ganar a Madrazo va a ser una ciudad del PRI, o por ejemplo anunciar más que son PRI, PRI, PRI".

José: "Por ejemplo, el gobernador en Monterrey, del PRI, en Monterrey, en Guadalupe, nomás han ganado en dos o en tres, y en diputación no sé; otro amigo de mi papá que quería ser y no pudo, como mi tío".

Escuela privada, 10 años.

Como señala Cullingford (1992), la televisión es considerada una fuente de información, sirve para enterarse de lo que sucede en la política y para ver lo que hacen los candidatos, incluso para decidir por quién se va a votar. Y de acuerdo a lo que reportan los niños de ambas escuelas, sus orientaciones están enlazadas a las orientaciones políticas y opiniones que tienen sus padres.

En la escuela privada, sobre todo en cuanto a la orientación de sus opiniones, y en la pública sobre todo en el tipo de consumo televisivo, 
como menciona Cullingford (idem) reconocen lo que ven en la televisión, pero no manifiestan delinear sus significados.

\section{DISTANCIAMIENTO CRÍTICO Y PERSUASIÓN DETECTADA}

La mayoría de los niños de la escuela privada identifican los spots publicitarios como comerciales, detectan una intención de persuasión por parte de los políticos y además expresan una actitud crítica ante lo presentado, lo cual tiene que ver con la guía de sus padres, tal como lo expresa Denisse:

- ¿Cómo platican de la política?

Denisse: "Pues cuando mi papá habla, habla de política, checamos los comerciales que hay de los presidentes y lo que dicen, y luego decimos si estamos de acuerdo o no. Mi papá nunca se pone de acuerdo con la mayoría de los seleccionados, de los que son candidatos para la presidencia, porque a veces sí son algo mentirosos, sí me he dado cuenta”.

Escuela privada, 10 años.

En cambio, en la escuela pública no se encontró ninguna respuesta en que reconocieran que los spots sobre temas políticos corresponden ya sea a un interés de información pública o a un orden con fines propagandísticos; sin embargo, quedan presentes en su memoria como las canciones de propaganda:

Ale: “¿En qué tipo de programas?” (te enteras de política)

Alejandra: "Yo, por la tele, del PRI de Canavati, vi uno que decía, era así de una cancioncita de Marcela Guerra, y un pacorrido en Hechos, no, pero hacían un comercial".

Ale: "¿Y el comercial cómo iba?"

Alejandra (canta): "Marcela... la voz de la gente, la amante de la gente, que viva Marcela, vota por ella,...yo soy Marcela Guerra y vamos por México hacia delante. Y luego sale otro, que dice: 'mira, ya no nos está alcanzando para el agua porque está aumentando mucho,' y otro señor dice: ‘¿cómo voy a pagar todo el recibo si la situación es desesperante?', y ahí estaba”.

Escuela pública, 12 años. 
La función de entretenimiento es reconocida por los niños e incluso propicia que quieran enterarse un poco más; también parecieran interesarse con el fin de entrar al mundo de los mayores. Consideran que enterarse de la política puede ser una buena opción para tener más opiniones y conversación con los padres.

Alejandra: "Como estamos los dos viendo, mi papá me platica de qué se está tratando y de repente pos me está interesando, y empiezo a verla junto con mi papá. Yo haz de cuenta que sale una noticia, ¿no? y pus yo la estoy viendo medio dormida desayunando y veo que se empieza a reír y yo le digo, ¿por qué te ríes? Y ya me empieza a explicar todo y al final empiezo a reírme. Nunca le entiendo a las noticias, aunque, bueno, cuando me explica mi papá ya les entiendo mejor".

Escuela privada, 12 años.

En ambos casos señalaron que no les gusta cuando, en las noticias, los políticos "hablan mucho" (Berenice, escuela pública). Los niños de ambas escuelas suelen definir la política en términos de las acciones políticas y de las elecciones. Muchos de los niños comentan sentirse ajenos a las noticias televisadas, sobre todo los niños de la escuela pública, ya que señalan no ver utilidad puesto que no pueden votar, lo que pudiera enfatizarse en tiempo electoral. Estos niños no mostraron actitudes cínicas, sin embargo se pudo observar que se sentían ajenos al tema político, desde la forma en que esta se presenta hasta la función, que ven lejanas a su vida cotidiana y práctica.

\section{LA DISCUSIÓN DE LA “POLÍTICA" Y EL MANEJO DE LO "POLÍTICO"}

Como se vio, tanto en el análisis cuantitativo como cualitativo, la figura del presidente es la más presente en la idea de los niños sobre la "política", y como decía López Gómez (2003) esto se debe quizás a que es la figura política más televisada y constante, no sólo en tiempos electorales. Hess y Torney (1967, en Fernández, 2005) remarcaban que los niños pequeños se centran en lo concreto, identificando por ejemplo al gobierno con el presidente, y Fernández (2005) señalaba que de acuerdo a la sociología política existe una idealización positiva de la figura presidencial. 
Los niños de la escuela privada entrevistada mostraron preferencia por el PAN, y las concepciones que tienen de los partidos son moderadas en su mayoría. Lo que coincide con lo reportado sobre su preferencia en caso de supuesto voto, en las encuestas, cuando se les preguntó que si pudieran votar por quién votarían, $50 \%$ de los encuestados señaló que por Felipe Calderón (PAN), siguiendo a Roberto Madrazo (PRI) con $20 \%$. Cullingford (1992) señala que la intención de voto está muy relacionada con sus creencias, como una cercana materialización a sus valoraciones políticas.

En el caso de la escuela pública, la mayoría de los niños definen positivamente a los políticos, pues señalan que en su mayoría son personas que ayuda a la ciudad. Coinciden en que votarían por el PRI, sólo José dijo que “...no, no, por López Obrador”, y con cierta duda. Esta concurrencia de respuesta y preferencia por algún partido coincide con lo reportado por los niños de escuelas públicas de Monterrey, ya que como lo reportan los datos cuantitativos de este estudio $42 \%$ votaría por Roberto Madrazo (PRI) y 28\% votaría por Felipe Calderón (PAN).

En la escuela pública se ve al presidente como una figura de autoridad que ha ayudado y que es cercano a ellos por el tema de Enciclomedia, como lo mencionaba Hiram (12 años, pública) "pues a él, la vez pasada lo vi que estaba anunciando sobre eso del pizarrón”, y dado que sale en televisión con niños, parece despertar cierta empatía.

Los niños tienen muy buena opinión de Fox, lo que coincide con lo que opinan los niños de la escuela privada, a pesar de que han escuchado rumores en torno a él, como menciona Berenice. En esta entrevista focalizada, menciona que Fox engañaba a las personas pobres y que las envenenaba, pero termina diciendo que también siente agrado por él, tal como lo expresaban las demás compañeras Nallela y Nancy; esto también se puede atribuir a un efecto de espiral del silencio, que por la presión social pueden incluso cambiar de opinión (Noelle-Neumann, 1995). Kohlberg (1964, en Fernández, 2005), mencionaba que niños entre 10 y 13 años inician una etapa en que intentan agradar a los demás.

Bere: "Este, así como este Fox piensan que va a ayudar a los pobres, que les da vitaminas, pero dicen que les dan para que se mueran para que haya menos, para que haya puro rico". 
Ale: “¿Eso en dónde lo escuchaste?”.

Bere: "Creo que en las noticias, o no me acuerdo".

Ale: "¿Y ustedes que piensan de su presidente?".

Bere: "Pues yo que está bien".

Nallela: "Pues no sé, a mí me cae muy bien Fox".

Nancy: "A mí también, a mí también me cae muy bien".

Ale: “¿Es bueno?”.

Todas: "Sí".

Bere: "Sí me gusta".

Escuela pública, 12 y 11 años.

Pese a una preferencia manifiesta por el PRI, los niños perciben como buena la imagen del presidente, y aunque puedan manifestar un desacuerdo por los rumores, manifiestan tener simpatía por él, lo que pudiera asociarse a lo que ya Rubén comentaba sobre el hecho de que lo ven saludando a los niños y por el programa Enciclomedia.

En ambas escuelas se observó un encuentro de opiniones distintas; sin embargo, en la escuela privada se llegó a un mayor planteamiento de argumentos en los puntos de vista, aun cuando parte de los fundamentos estuviera en el hecho de que se vio en televisión, para explicar su punto. En la escuela pública se recurrió a un supuesto, y para llegar a un acuerdo se apeló a la simpatía que sentían por el personaje político.

En estas conversaciones podemos ver que, como menciona Orozco (2001), las condiciones del entorno social son las que dan sentido a su cultura política particular y a lo que de ella exponen. Pero no siempre se trata de una aceptación o rechazo cognitivo del mensaje, muchas veces están implicadas otras condiciones en relación con su contexto cultural y con otras herramientas más allá de la asimilación cognitiva. En ocasiones, las emociones y sentimientos se involucran en un plano distinto, coincidiendo con lo que señalaba Greenstein (1965, en Fernández, 2005), en cuanto a que en el desarrollo político del niño existe un predominio de lo afectivo sobre lo cognitivo, lo que propicia un desarrollo de actitudes, primordialmente, antes que de conocimientos.

Bourdieu (en Flashsland, 2005) dice que hay que descubrir lo arbitrario y la contingencia allí donde se quiere ver la necesidad o la natu- 
raleza, y descubrir la necesidad o coacción social donde se quiere ver la elección y el libre arbitrio.

Este estudio pretendió dar un acercamiento al lugar que ocupa la política televisada en las opiniones y percepciones de los niños y comprender, a partir de lo que los niños reportaron en cuestionarios y entrevistas, hasta qué punto es distinto su acercamiento de acuerdo al tipo de escuela a la que pertenecen y del capital cultural heredado de sus padres.

La investigación se enfocó en el fenómeno de recepción desde la brecha educativa en nuestro país, a fin de identificar cómo la estructura educativa está también involucrada en la brecha de capacidades interpretativas, reflexivas y críticas que pudieran motivar una mejor ciudadanía y un reparto del poder mucho más equilibrado.

Las audiencias construyen su opinión pública, son diversas y realizan apropiaciones variadas de acuerdo a sus condiciones y herramientas de interpretación. Los niños, ciudadanos en formación, están percibiendo la información televisada de la política; la formación está prioritariamente orientada por sus padres y la escuela en la que se encuentran.

\section{DISCUSIÓN}

En este estudio se pretendió conocer qué factores están conformando la cultura política de los niños regiomontanos a partir de la campaña presidencial 2006, a fin de tener una mejor idea sobre sus percepciones, actitudes y opiniones de la política, cómo se involucran o no con ella, y el tipo de apropiaciones que hacen de esta información televisada.

A diferencia de los adolescentes ingleses observados por Buckingham (2003), los niños de Monterrey no mostraron actitudes cínicas, lo que puede estar relacionado con diversos factores como la edad y sus implicaciones emocionales, el contexto cultural, social y político propios del país. Buckingham argumenta, además, que una de las razones de estas actitudes puede estar asociada a una creciente individualidad en la cultura política londinense, que también suele detectarse en el estudio de Cullingford (1992). En el caso mexicano, no se ha hablado mucho sobre si los niños se están orientando al individualismo, pero estudios como el de Monsiváis (2002) sí parecen indicar que la sociedad 
y el tejido social tienden al razonamiento individualista al menos en el caso de los jóvenes.

En el presente estudio se pudo identificar en los niños de la escuela pública cierta ingenuidad ante la información política televisada, pues no parecen cuestionar las intenciones de los políticos cuando presentan sus spots ni cuestionar más allá la información procedente de los noticiarios. Tienden a asumirlas acríticamente, derivando sus opiniones y valoraciones, sobre todo desde lo emocional y empático, no desde la guía de sus padres, sólo en la orientación del consumo televisivo. No se puede establecer que estén siendo influidos por el consumo televisivo; además, solían tener un consumo más relacionado con sus emociones, como son las noticias "de tragedias". Sin embargo reportaron mejores actitudes hacia la política, más valoración en cuanto a la participación política, y a la política en general, que los niños de la escuela privada.

La brecha educativa parece generar audiencias que están formando su opinión pública desde sus recursos y referentes del entorno cultural, una apropiación distinta que, como señalaban Croteau y Hoynes (2003), pudiera estar haciendo visibles ciertas cosas para un grupo, pero no de la misma manera para el otro.

Los niños de las escuelas privadas tienen padres con más preparación que los niños de la escuela pública, mayor capital cultural, mayor atención y transmisión en las redes de comunicación interpersonal que moldean la recepción masiva y posibilitan percepciones profundas.

De acuerdo con varios estudios (Friedamann, 1997, en Fernández 2005), las personas con una mayor educación presentan determinadas características como la disposición a participar en la política, un sentimiento pronunciado a favor de la valoración de la eficacia política, mayor compromiso con las organizaciones políticas y el proceso de toma de decisiones; son más conscientes de la influencia y efectos que la política tiene para el individuo, dan mayor seguimiento a los acontecimientos políticos y se suelen comprometer con mayor facilidad en las discusiones políticas. Algunas de estas características las vemos proyectadas en los niños de la escuela privada.

Orozco (1999) mencionaba que en la recepción podía darse la manipulación o emancipación de la audiencia. En la investigación se encontró que la emancipación pudiera estar lejana, ya que en casos 
de valoraciones participativas pareciera que esto se ligara más a una ingenuidad, como consecuencia de un consumo mediático acrítico y por la poca orientación por parte de los padres, como se reportó en la escuela pública.

Por otro lado, el panorama en la escuela privada tampoco pareciera ser alentador, pues según los reportes del estudio, los niños no suelen valorar la participación y parecen estar más apáticos a involucrarse políticamente, en este caso, quizá, hasta como una derivación de una actitud crítica y analítica de la política televisada y de la orientación que reciben de sus padres. En este sentido, Fernández (2005) dice que la socialización política es un proceso casi siempre conservador, puesto que ayuda a perpetuar el statu quo, y la televisión parece el referente idóneo para este estado.

La preferencia por las noticias locales no es igual en todas las zonas del país; en Monterrey sí se puede establecer una constancia de la preferencias por la televisión local, como lo muestra el estudio de Lozano (2000), quien encontró que en adultos de distintas clases sociales es constante la preferencia por noticieros locales, no sucediendo lo mismo en otro estudio (Orozco, 2001) llevado a cabo en León, Guanajuato y ciudad de México, donde las noticias locales no interesaban y parecían inútiles.

Buckingham (2003) señala que altos niveles de uso de noticias televisivas se correlacionan con altos niveles de participación política, aunque la influencia de los padres, los compañeros o factores comunitarios, son reconocidos como más significativos.

Berger (2002) observa cómo se involucran las influencias culturales, por ejemplo el consumo y entorno cultural que pudieran tener los niños en su proceso de socialización, que los llevarán a interactuar colectiva o individualmente. A partir de lo que Berger observó, podemos concluir que la interacción con la política puede estar más presente en los niños de la escuela privada y, sin embargo, la movilización política pudiera ser más frecuente en los niños de la escuela pública, ya que tienen más inquietudes de participación.

En Monterrey podría sugerirse que hay dos comunidades de apropiación a partir de las condiciones culturales y socioeconómicas de los niños y de las herramientas educativas que están o no a su alcance. En el plano educativo, podemos señalar que no sucede de la misma forma en 
otros países. En México se sigue reportando una brecha educativa preocupante; sin embargo, como se analizó, el papel de la figura paterna es crucial, así como la herencia cultural que aporte al niño.

Buckingham (2003) afirma que el sentido de alienación que los niños tienen frente a la política no puede ser vista simplemente como un tipo de desinterés, apatía e ignorancia. Al contrario, debe ser vista como una respuesta lógica a las construcciones de nuestra posición social. Buckingham reconoce que hay cierta ceguera a distinguir realidades de otros modos de hacer y percibir.

Flores (2000) señala que uno de los nexos indispensables entre el ciudadano y la democracia es el que se establece por medio de la educación, junto con los accesos a la información política, que han sido considerados como garantía del buen ejercicio de la ciudadanía.

Este estudio también abre la posibilidad de análisis posteriores, tomando en cuenta los cambios de acuerdo a edades, tiempos políticos, ciudades y regiones, a fin de poder entender más sobre el fenómeno de la recepción de la política televisada en distintos entornos y sus consecuencias, propiciando una comunicación política más democrática.

Un buen principio será, como menciona Flores (idem), mejorar la idea en el mexicano de que la información política puede ser un buen parámetro para evaluaciones políticas y posibilitar el desarrollo de capacidades de reflexión, como lo han propuesto autores como Orozco para una educación de la recepción. Es decir, tener criterios para hacer de esa información un conocimiento reflexivo y crítico. Y un buen principio será reconocer la importancia de los niños, escucharlos y verlos como parte del proyecto social, dándoles también su lugar como sujetos políticos. Como se señaló en el planteamiento del problema, en las nuevas generaciones está la posibilidad de reforzar el tejido social y posibilitar mejores respuestas sociales ante los problemas políticos.

\section{Bibliografía}

ANG, I. (1991) "Wanted audiences: on the politics of empirical audience studies", en E. Seiter, H. Borchers, G. Kreutzner y E.M. Warth (Eds.), Remote control: television, audiences \& cultural power. Londres: Routledge, pp. 96-115. 
BOURDIEU, P. (2000) La distinción. Taurus: España.

BUCKINGHAM, D. (2000) The making of citizens: young people, news and politics. Londres y Nueva York: Routledge.

CALleS, J. (1999) "Recepción, cultura política y democracia”. Comunicación y Sociedad 36, pp. 47-69. UdeG: México.

CHAFFE, S. y Yang, S. (1990) “Communication and political socialization", en O. Ichilov (ed), Political socialization, citizenship education and democracy. Nueva York: Teachers College Press.

CHARLES, M y Orozco, G. (1992) Educación para los medios. Una propuesta integral para maestros, padres y niños. México: ILCE/ UNESCO.

CROTEAU, D. y Hoynes, W. (2003) Media/society: industries, images, and audiences. Thousand Oaks: Pine Forge Press.

CullingFORD, C. (1992) Children and society. Children's attitudes to politics and power. Londres y Nueva York: Casell.

Delahanty, G. (1995) "Psicología política en el México de hoy”, en González, M y Delahanty, G. (ed.), Psicología política y socialización de la representación ideológica, pp. 211-217. México: Universidad Autónoma Metropolitana

ENCUP (2001) Encuesta nacional sobre cultura política y prácticas ciudadanas. Mayo, 15, 2007. Disponible en: http:/www.inegi.gob. $\mathrm{mx} /$ est/contenidos/espanol/proyectos/metadatos/encuestas/encppc_ 239.asp? $\mathrm{c}=4887$.

ESTEINOU, J. (2003) "Siglo XXI, leyes del mercado y nuevo proyecto de comunicación en América Latina”. Texto Abierto, 3 (3/4), pp. 127-178.

EvelAND, W., McLeod, J., Horowitz, E. (1998) "Communication and age in childhood political socialization: an interactive model of political development". Journalism and Mass Communication Quarterly, 75 (4), pp. 699-719.

FERNÁNDEZ, A. (2005) Infancia, adolescencia y política en México. Distrito Federal: Instituto Electoral del Distrito Federal.

FISKE, J. (1987) Television Culture. London: Methuen y Co. Ltd.

FLACHSLAND, C (2005) Pierre Bordieu y el capital simbólico. España: Campo de ideas.

FLORES, J., Meyenberg, Y. (2000) Ciudadanos y cultura de la democracia. Consultado en septiembre, 5, 2006 en www.ife.mx. 
GARCÍA, N. (2004). Diferentes, desiguales y desconectados. Barcelona: Gedisa.

GONZÁLEZ, J. (2001) La cultura en México. México: Consejo Nacional para la Cultura y las Artes, Dirección General de Culturas Populares: Programa Cultura, Centro Universitario de Investigaciones Sociales.

GUNTER, B. y McAleer, J. (1997) Children and television. Nueva York: Routledge.

HALL, S. (2001) "Encoding/decoding”, en M. G. Dirham y D. M. Kellner (eds.), Media and cultural studies: keyworks, pp. 166-176. Malden, MA, EUA: Blackwell Publishing.

IBARRA, A. (2003) Televisión y socialización política de escolares en la zona metropolitana de Guadalajara (tesis doctoral, Universidad de Guadalajara).

IFE (2006) Reporte final de los monitores de promocionales 2006. Consultado en febrero, 26, 2007. Disponible en: www.ife.org.mx/docs/ internet/IFE_Home/CENTRAL/Contenidos_Centrales/estaticos/ Monitoreo/rep_final_monitoreos.pdf

IgARTUA, J. J. (2006) Métodos cuantitativos de investigación en comunicación. Barcelona: Bosch.

JARA, R (2007) ¿Cómo la ves? La televisión mexicana y su público. IBOPE. México

JENSEN, K. (1992) "Hablan los televidentes. Estudios de recepción en varios países”, en Orozco, G. (ed.), La política del multisignificado. Noticias en la televisión, conciencia cotidiana y acción política, pp. 97-130. México: Universidad Iberoamericana.

KIM, J., Wyatt, R. y Katz, E. (1999) “News, talk, opinion, participation: the part played by conversation in deliberative democracy". Political Communication, 16 (4), pp. 361-385.

KIOUSIS, S., McDevitt, M.y Wu, X. (2005) "The Genesis of Civic Awareness". Journal of Communication, 12 (3), pp. 756-774.

LIEBES, T. y Katz, E. (1989) "On the critical abilities of television viewers”, en Seiter, E., Borchers, H., Kreutzner, G. y Warth, E.M. (Eds.), Remote control: television, audiences and cultural power, pp. 204-222. Londres: Routledge.

LINDLOF, T. (1995) Natural audiences: qualitative research of media uses and effects. Sage: Estados Unidos. 
LÓPEz Gómez, G. (2003) Televisión y socialización politica de los niños en Monterrey y Juchitán, (tesis de maestría, Tecnológico de Monterrey, Monterrey), México.

LOZANO, J. C. (2003) "Distanciamiento crítico frente a la TV nacional mexicana". ZER, 4, pp. 131-149.

- (2000) "El género y el nivel socioeconómico como mediaciones en el consumo de noticieros televisivos en México". Revista de Estudios de Comunicación ZER, pp. 1-19.

- (1991) "Del imperialismo cultural a la audiencia activa: aportes teóricos recientes". Comunicación y Sociedad, 10-11, pp. 85-106, México: Universidad de Guadalajara.

LULL, J. (1997) Medios, comunicación, cultura: aproximación global. Buenos Aires: Amorrortu.

MCCOMBS, M. (2006) El establecimiento de la agenda. El impacto de los medios en la opinión pública y en el conocimiento. España: Paidós Comunicación.

MEIRICK P., Wackman D. (2004) “Kids voting and political knowledge: narrowing gaps, informing votes”. Social Science Quarterly, 85 (5), pp. 1161-1214.

MONSIVÁIS, A. (2002) La democracia ajena. Jóvenes, socialización política y constitución de la ciudadanía en Baja California. Center For Us-Mexican Studies.

MORLEY, D. (1992) "Interpreting television: the nationwide audience", en Television, audiences and cultural studies, pp. 75-118. Londres y Nueva York: Routledge.

MOUFFE, C. (1999) El retorno de lo político: comunidad, ciudadanía, pluralismo, democracia radical. Barcelona: Paidós.

NATERAS, O. (1995). Psicología política en el México de hoy. En González, Delahanty. México: UAM Iztapalapa.

NOELLE-Neumann, E. (1995) La espiral del silencio. Barcelona: Paidós. OCDE (2007) "Mucho gasto y pobres resultados en educación básica en México" (noviembre 22). Disponible en: La Jornada, http://www. jornada.unam.mx/ultimas/2007/11/22/mucho-gasto-y-pobres-resultados-en-educacion-basica-en-mexico-ocde.

Orozco, G. (2001) Televisión, audiencias y educación. México: Buenos Aires: Norma. 
- (1996) Televisión y audiencias: un enfoque cualitativo. Madrid: De la Torre.

- (1992) "Hablan los televidentes, estudios de recepción en varios países", en Orozco, G. (ed.), Televisión y educación en México, pp. 11-32. México: Universidad Iberoamericana.

RENERO, M. (1992) "De géneros televisivos y usos familiares". Comunicación y Sociedad, 3, pp. 19-38. Nueva Época, México: Universidad de Guadalajara.

RoBLes, Y. (2003) Mensajes televisivos y discurso infantil sobre desigualdades sociales en dos grupos sociales de la zona metropolitana de Guadalajara (tesis de maestría, Universidad de Guadalajara).

RoldÁn, N. (2007) Privadas, nueve de las diez mejores primarias: SEP. El Norte, $22 \mathrm{pp}$.

SÁNCHEZ, R. (1992) "Educación, medios de difusión y democracia". Estudios sobre las culturas contemporáneas, 4 (13-14), pp. 29-56.

SEARS, D. y Valentino, N. (1998) "Politics matters: political events as catalysts for preadult socialization". American Political Science Review, 91, pp. 45-65.

SECRETARÍA de Gobernación (2005) Encuesta Nacional de Cultura Política de 2005. Consultado en septiembre, 12, 2006. Disponible en: http://www.gobernacion.gob.mx/index.php

SEGOB (2002) "Conociendo a los ciudadanos mexicanos: principales resultados de la Encuesta Nacional sobre Cultura Política y Prácticas Ciudadanas 2001”. Este País, (137), pp. 1-24.

SEGOVIA, R. (2001) La politización del niño mexicano. México: El Colegio de México.

TAPIA, E. (2003) Socialización política y educación cívica en los niños. Querétaro, México: Instituto Mora.

TAYLOR, S. y Bogdan, R. (1998) Introducción a los métodos cualitativos de investigación : la búsqueda de significados. Barcelona: Paidós.

WeINTRAUB, E. y Pinkleton, B. (2001) "The role of parental mediation in the political socialization process". Journal of Broadcasting and Electronic Media, 45 (2), pp. 221-241. 